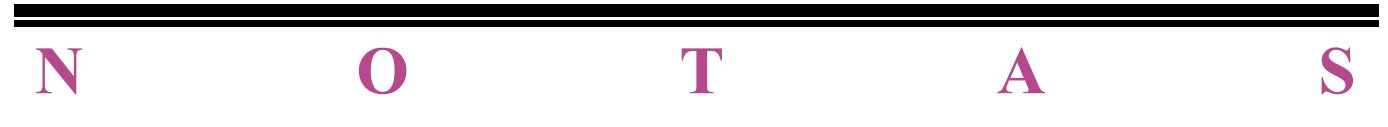

$\begin{array}{lllllllllllll}S & O & B & R & E & & L & A & & R & E & G & L\end{array}$

$\begin{array}{llllllllllllllll}D & E & R & E & C & O & N & O & C & I & M & I & E & N & T & O\end{array}$ 


\section{ALGUNAS CONSIDERACIONES SOBRE LOS SISTEMAS JURÍDICOS}

\section{Sistemas normativos estáticos}

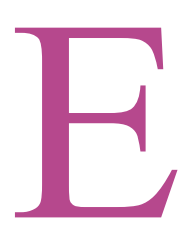

s casi un lugar común del pensamiento jurídico, que las normas jurídicas que integran el derecho de un país constituyen un conjunto unitario al que cabe atribuir carácter de sistema. Corresponde a la filosofía jurídica elucidar el concepto o los conceptos de sistema que usan los juristas. No debe extrañar, pues, que casi todos los filósofos del derecho, desde Bentham y Austin hasta Kelsen y Hart, hayan dedicado gran parte de sus esfuerzos a este tema, que constituye uno de los problemas centrales de la filosofía jurídica moderna. No obstante los esfuerzos realizados, quedan todavía muchos puntos oscuros y no pocos problemas sin resolver. En este trabajo me propongo analizar algunos aspectos de esta problemática.

En Alchourrón-Bulygin 1971 hemos tratado de elaborar un modelo analítico de sistema como instrumento conceptual operativo para dar cuenta de algunos aspectos de la ciencia jurídica. Utilizando la noción de sistema deductivo elaborado por Tarski (1956) el sistema jurídico fue definido como un conjunto de enunciados jurídicos (que constituyen la base axiomática del sistema) que contienen todas sus consecuencias. Para que un sistema tal sea normativo, los enunciados de la base deben contener por lo menos algunos enunciados normativos o normas. Normas son definidas, a su vez, como enunciados que correlacionan ciertas circunstancias fácticas (casos) con determinadas consecuencias jurídicas (soluciones). Pero no es necesario que todos los enunciados de la base sean normativos: de hecho en los textos jurídicos (códigos, leyes, etc.) se encuentran a menudo al lado de normas enunciados de otro tipo, por ejemplo, definiciones (cfr. Alchourrón-Bulygin, 1982). Con esta reserva, hablaré en lo sucesivo, por razones de brevedad, de normas, entendiendo por tales enunciados jurídicos, sean éstos normativos o no. En el modelo 
las normas son enunciados con un significado definido, lo que permite eludir la vieja discusión de si las normas son entidades lingüísticas (enunciados u oraciones de cierto tipo) o significados o sentidos de tales enunciados (cfr. Bulygin, 1986).

Buena parte del trabajo de la ciencia jurídica (especialmente de la llamada dogmática jurídica) consiste en desplegar la base axiomática elegida, determinando qué soluciones corresponden a los distintos casos, lo que presupone la determinación previa del conjunto de todas las circunstancias fácticas o casos posibles (Universo de Casos) y del conjunto de todas las soluciones admisibles (Universo de Soluciones). De esta manera puede determinarse si el sistema es completo en el sentido de que resuelve todos los casos posibles o si, por el contrario, contiene algunas «lagunas normativas», es decir, casos a los que las normas no correlacionan ninguna solución. También puede establecerse si el sistema es coherente en el sentido de que en ningún caso hay conflictos entre normas o contradicciones normativas y también si las normas del sistema son independientes entre sí o redundantes en algún caso. De este modo el modelo permite definir con precisión ciertas propiedades estructurales de los sistemas jurídicos (completitud, coherencia e independencia) y, por consiguiente, dar cuenta de algunos aspectos importantes de la actividad típica de los juristas dogmáticos, a la vez que resolver el viejo problema de las lagunas del derecho.

Esta definición de «sistema jurídico» no prejuzga sobre el número y el origen de las normas que se elijan como base axiomática; puede tratarse de normas legisladas o consuetudinarias, extraídas de sentencias judiciales o de escritos doctrinarios y puede tomarse como base del sistema cualquier conjunto de normas: algunos artículos de un código o de una ley, todas las normas referentes a una determinada materia e incluso todas las normas pertenecientes al derecho de un país. Este último es un caso límite que ofrece algún interés (cfr. infra, sección V).

Dado que el sistema está definido como un conjunto de normas, éstas permanecen fijas en el modelo: cualquier cambio de la base axiomática del sistema nos llevaría a otro sistema, distinto del anterior. En este sentido, el concepto de sistema elaborado en Normatives Systems es estático. (Creo que esta noción de un sistema estático reconstruye con bastante fidelidad lo que Kelsen entiende por tales sistemas). Pero cuando los juristas hablan de sistema jurídico quieren dar cuenta, a menudo, del fenómeno del cambio: las normas jurídicas suelen cambiar con el transcurso del tiempo; algunas normas existentes son eliminadas, otras modificadas y también suelen agregarse normas totalmente 
nuevas. La posibilidad de tales cambios temporales determina el carácter dinámico del derecho. Para dar cuenta de este carácter es menester elaborar un concepto dinámico del sistema.

\section{Orden jurídico como sistema dinámico}

Si el sistema jurídico se concibe como un conjunto de objetos de cierto tipo (por ejemplo, un conjunto de normas o de enunciados jurídicos) y el conjunto es definido extensionalmente, entonces el sistema tiene que estar referido a un punto temporal determinado, pues con todo cambio provocado por los actos de creación o de derogación de normas, el conjunto deja de ser el mismo y se convierte en otro distinto, con lo cual volvemos a tener un concepto estático de sistema. Por lo tanto, un sistema dinámico no puede ser un conjunto de normas, sino una familia (es decir, un conjunto) de conjuntos de normas, o más precisamente una secuencia temporal de conjuntos de normas. Esto nos ha llevado a proponer una distinción terminológica entre sistema jurídico como conjunto de normas y orden jurídico como secuencia de sistemas jurídicos (Alchourrón-Bulygin, 1976). Desde entonces esta terminología parece haber adquirido una firme carta de ciudadanía en la literatura especializada (cfr. Caracciolo, 1988).

Los términos «sistema» y «orden» apuntan a la misma distinción conceptual que «sistema momentáneo» y «sistema jurídico» introducidos por Raz (1970). Sin embargo, Raz parece entender por «sistema momentáneo» la totalidad de las normas jurídicas que forman parte del derecho de un país en un momento temporal determinado; en cambio, el concepto de sistema caracterizado más arriba es más flexible, ya que su base puede estar formada por cualquier conjunto de enunciados jurídicos, lo que permite acercarse más a la forma en que trabajan los juristas, a quienes sólo en situaciones muy especiales interesa construir un sistema global formado por todas las normas de un derecho nacional. El sistema global es un caso particular, aunque obviamente importante, pues los juristas exigen a veces que la base de su sistema esté formada por algún subconjunto de las normas válidas, es decir, pertenecientes al derecho de ese momento. (Más adelante veremos que esta exigencia es mucho menos frecuente de lo que suele creerse: cfr. infra. sección V.) Y para saber si la base del sistema cumple este requisito es necesario poder identificar el sistema global. Por otra parte, el término «momentáneo» 
no es muy adecuado, pues insinúa que el sistema tiene una duración muy corta, cuando en realidad el sistema es relativo a un intervalo temporal entre dos actos de creación y/o derogación normativa (cfr. Bulygin, 1982) y este intervalo, sobre todo en el caso de sistemas formados por unas pocas normas, puede ser relativamente largo.

Por otra parte, no es claro si por «sistema jurídico» Raz entiende un conjunto de sistemas momentáneos o un conjunto de normas, a saber, todas las normas que pertenecen a todos los sistemas momentáneos que corresponden al sistema jurídico en cuestión. Si el sistema jurídico es un conjunto de sistemas momentáneos, la relación entre un sistema momentáneo y el sistema jurídico es la de pertenencia; si es un conjunto de normas y los sistemas momentáneos son subclases del sistema jurídico, entonces la relación es de inclusión. La terminología de Raz es vacilante en este punto. Por un lado habla de pertenencia («The phrase "The English legal system at the beginning of the reign of Elizabeht II" is ambiguous. It may refer to the momentary system of that particular time or to the legal system to which this momentary system belongs.» Raz, 1970, pág. 35; el subrayado es mío), por el otro, dice en la misma página que un sistema momentáneo es una subclase de un sistema jurídico. De todos modos, el concepto interesante es el del sistema jurídico como el conjunto de todos los sistemas momentáneos y no como el conjunto de todas las normas de todos los sistemas momentáneos, pues este último conjunto sería irremediablemente inconsistente, ya que contendría todas las normas derogadas y las que fueron dictadas en su lugar. En este sentido la distinción propuesta («sistema jurídico», vs. «orden jurídico») parece preferible, tanto desde el punto de vista conceptual, como terminológico.

\section{Identidad y estructura del orden jurídico}

Si se acepta la distinción entre orden jurídico y sistema jurídico se plantean dos problemas mayores, que Caracciolo llama problema de identidad y problema de estructura (cfr. Caracciolo, 1988). El primero es el de la determinación de la identidad de un orden jurídico, es decir, la cuestión de saber cuándo una secuencia de sistemas pertenece al mismo orden jurídico y cuándo se interrumpe esa secuencia y surge un nuevo orden jurídico, distinto del anterior. Para ello es necesario explicitar los criterios de pertenencia de los sistemas a un orden jurídico.

El segundo problema consiste en determinar el contenido de 
un sistema jurídico global correspondiente a un determinado intervalo temporal y para lo cual tenemos que conocer los criterios de pertenencia de las normas al sistema en cuestión. En cierto sentido ambos problemas se refieren a la identidad: la identidad del orden jurídico y la identidad de un sistema jurídico, y ambos consisten en la determinación de los criterios de pertenencia: pertenencia de las normas al sistema y pertenencia de los sistemas al orden. Sin embargo, hablar del problema de estructura tiene su razón de ser, pues los criterios de pertenencia de las normas al sistema determinan la estructura de este último.

Caracciolo distingue dos criterios de pertenencia que considera básicos: criterio de deducibilidad $\left(\mathrm{C}_{1}\right)$ y criterio de legalidad $\left(\mathrm{C}_{2}\right)$. De acuerdo al criterio de deducibilidad una norma pertenece al sistema cuando es consecuencia lógica (es deducible de) las normas pertenecientes al sistema. Según el criterio de legalidad una norma pertenece al sistema si ha sido creada (promulgada) por una autoridad competente del sistema. (Una autoridad x es competente para dictar o promulgar una norma $\mathrm{N}$, cuando existe una norma que pertenece al sistema que le otorga competencia, es decir, le autoriza a $\mathrm{x}$ a dictar la norma N.) Esto permite distinguir entre dos relaciones entre las normas del sistema, relación de implicación y relación de legalidad. Estas relaciones determinan la estructura del sistema.

Las distintas formas de combinación de estos dos criterios permiten a Caracciolo distinguir entre cuatro modelos analíticos de pertenencia: $M_{1}$ basado en $C_{1} M_{2}$ basado en $C_{2}$ $\mathrm{M}_{3}$ basado en la conjunción de los dos criterios $\mathrm{C}_{1} \mathrm{y} \mathrm{C}_{2}$ y $\mathrm{M}_{4}$ basado en la disyunción de $\mathrm{C}_{1}$ $\mathrm{yC}_{2}$.

De estos cuatro modelos $\mathrm{M}_{4}$ en el cual ambos criterios, el de deducibilidad y el de legalidad constituyen condiciones suficientes, aunque no necesarias para la pertenencia, es decir, una norma pertenece al sistema, sea porque se deduce de las normas del sistema, sea porque ha sido dictada por una autoridad competente, es el que mejor reconstruye los criterios de pertenencia que los juristas usan efectivamente, aunque bien puede ser que tales usos no sean homogéneos, en el sentido de que respecto de alguna subclase de normas el modelo aplicable sea otro. En particular, Caracciolo sugiere que en el caso de las sentencias judiciales el modelo más adecuado es $\mathrm{M}_{3}$ esto es, el que exige el cumplimiento de ambos criterios, lo que equivaldría a decir que los jueces sólo son competentes para promulgar normas que son consecuencia lógica de las normas generales del sistema (más la descripción del caso). Esto suena bastante razonable, y es un argumento en favor de no mezclar las normas generales con las 
normas individuales. En otras palabras, opino que el sistema jurídico (y, por ende, el orden jurídico) ha de ser reconstruido de tal manera que su base sólo incluya normas generales y no las normas individuales, como, por ejemplo, las sentencias judiciales. La descripción completa del derecho vigente en un momento dado no incluiría seguramente tales normas individuales; una especificación completa de las normas generales vigentes en el momento requerido sería considerada, sin duda, como una descripción satisfactoria. Por estas razones considero conveniente limitar la base del sistema a normas generales; las normas individuales formarán parte del sistema sólo a título de consecuencias lógicas en virtud del principio de deducibilidad.

Los criterios de deducibilidad y de legalidad son manifiestamente insuficientes para dar cuenta de la pertenencia de todas las normas del sistema, pues presuponen que el sistema ya tiene normas, cuya pertenencia no depende de ninguno de los dos criterios. Siguiendo la terminología de Caracciolo podemos llamar independientes a tales normas.

Por otra parte es interesante la observación de Caracciolo de que las normas que se incorporan conforme al criterio de legalidad son dependientes de las normas del sistema anterior; lo mismo ocurre con la eliminación de las normas como consecuencia de la derogación. De esta manera el criterio de legalidad constituye, a la vez, un criterio para la pertenencia de un sistema al orden jurídico. Como dice Caracciolo: «...un sistema normativo $\mathrm{S}_{\mathrm{t}}$ pertenece al orden jurídico $\mathrm{O}_{\mathrm{i}} \mathrm{si}$, y sólo si: a) las normas inéditas de $\mathrm{S}_{\mathrm{t}}$ con respecto al contenido del sistema anterior $\mathrm{S}_{\mathrm{t}-1}$ que pertenece a $\mathrm{O}_{\mathrm{i}}$ han sido emitidas mediante actos autorizados por normas que pertenecen a $S_{t-1} ; b$ ) las normas de $S_{t-1}$ que no pertenecen a $S_{t}$ han sido derogadas por actos autorizados por normas de $\mathrm{S}_{\mathrm{t}-1}$. La aplicación de ambos requisitos asegura, entonces, la legalidad del cambio temporal de sistemas pertenencientes al orden jurídico» (Caracciolo, 1988, 68). El requisito a) no significa que todas las normas de $\mathrm{S}_{\mathrm{t}}$ tengan que haber sido promulgadas de acuerdo a $\mathrm{S}_{\mathrm{t}-1}$ sino sólo las «nuevas» o «inéditas», es decir, las que no pertenecen al sistema anterior $S_{t-1}$.

Lo dicho implica que todo orden jurídico tiene que originarse en un sistema de normas independientes; esas normas, salvo que sean derogadas o modificadas en el futuro por autoridades competentes, pertenecen a todos los sistemas subsiguientes de ese orden. Todos los sistemas pertenecientes a ese orden, salvo el primero, se originan a partir del sistema inmediatamente anterior mediante adición de normas nuevas (como consecuencia de actos de promulgación o creación normativa) o por medio de la 
sustracción de normas (como consecuencia de actos de derogación) y, en última instancia, a partir del sistema originario de normas independientes.

Desde luego, tanto los actos de promulgación como los de derogación tienen que ser llevados a cabo por autoridades competentes, es decir, tienen que cumplir con el criterio de legalidad $\mathrm{C}_{2}$. En este sentido las normas independientes originarias constituyen la base del orden jurídico. El conjunto de las normas independientes que constituyen la base de un orden Jurídico $\mathrm{O}_{\mathrm{i}}$ será denominado -siguiendo la terminología de Kelsen- la primera constitución de ese orden jurídico.

Para poder hablar de un orden jurídico hay que partir de una primera constitución, esto es, de un conjunto de normas que se decide adoptar como base del orden. Este conjunto se identifica extensionalmente, es decir, por enumeración: $\left[\mathrm{N}_{1}, \mathrm{~N}_{2} \ldots \mathrm{N}_{3}\right]$. Las normas contenidas en la primera constitución y todas sus consecuencias lógicas forman un sistema normativo, el primero de una serie temporal de sistemas. Pero para que haya una serie temporal tal, la constitución tiene que contener al menos una norma de competencia que faculte a una autoridad a promulgar nuevas normas y, eventualmente, a derogar normas existentes. El segundo sistema de la serie surge en el momento cuando una autoridad competente de acuerdo al primer sistema promulgue una nueva norma y/o cuando derogue alguna norma. De ahí se desprende que a todo intervalo temporal entre dos actos normativos corresponde un sistema y que todo acto de promulgación y todo acto de derogación (realizados por una autoridad competente) dan lugar a un nuevo sistema que integra la serie. La modificación o reforma de una norma puede ser analizada como derogación y promulgación simultáneas: nada impide, por cierto, que una norma sea derogada y simultáneamente se promulgue otra en su lugar.

De lo dicho se sigue que todo orden jurídico tiene por definición una primera constitución. Esta constitución puede, desde luego ser modificada total o parcialmente, dando lugar a nuevas constituciones (derivadas), siempre que se establezca en ella algún procedimiento de reforma.

\section{Criterios de identificación}

Estamos en condiciones de formular el siguiente criterio de identificación de un orden jurídico determinado $\mathrm{O}_{\mathrm{i}}$ :

1. El conjunto de normas $\left[\mathrm{N}_{1}, \mathrm{~N}_{2} \ldots \mathrm{N}_{3}\right]$ es el sistema originario (primera constitución) de $\mathrm{O}_{\mathrm{i}}$. 
2. Si una norma $N_{j}$ es válida en un sistema $S_{t}$, que pertenece a $O_{i}$ y $N_{j}$ faculta a la autoridad $x$ a promulgar la norma $N_{k}$ y x promulga en el momento t la norma $N_{k}$, entonces $N_{k}$, es válida en el sistema $S_{t+1}$ (es decir, en el sistema correspondiente al momento siguiente a t) y $S_{t}$ pertenece a $\mathrm{O}_{\mathrm{i}}$.

3. Si una norma $\mathrm{N}_{\mathrm{j}}$ es válida en un sistema $\mathrm{S}_{\mathrm{t}}$, que pertenece a $\mathrm{O}_{\mathrm{i}}, \mathrm{y} \mathrm{N}_{\mathrm{j}}$ faculta a la autoridad $\mathrm{x}$ a derogar la norma $\mathrm{N}_{\mathrm{k}}$, que es válida en $\mathrm{S}_{\mathrm{t}} \mathrm{y}$ x deroga $\mathrm{N}_{\mathrm{k}}$ en el momento $t$, entonces $\mathrm{N}_{\mathrm{k}}$ no es válida en el sistema $\mathrm{S}_{\mathrm{t}+1}$ (correspondiente al momento siguiente a $\mathrm{t}$ ) que pertenece a $\mathrm{O}_{\mathrm{i}}$.

4. Las normas válidas en un sistema $S_{t}$ que pertenece a $O_{i}$ que no han sido derogadas en el momento $t$, son válidas en el sistema $\mathrm{S}_{\mathrm{t}+1}$ de $\mathrm{O}_{\mathrm{i}}$ (que corresponde al momento siguiente a t).

5. Todas las consecuencias lógicas de las normas válidas en un sistema $\mathrm{S}_{\mathrm{t}}$ que pertenece a $\mathrm{O}_{\mathrm{i}}$ también son válidas en $\mathrm{S}_{\mathrm{t}}$.

(Para evitar excesivas repeticiones del término «pertenece», uso la frase «la norma $\mathrm{N}$ es válida en el sistema $\mathrm{S}$ » como sinónimo de «la norma $\mathrm{N}$ pertenece al sistema $\mathrm{S}$ » $\mathrm{y}$ reservo el término «pertenencia» para referirme a la relación entre un sistema y un orden.)

El criterio de identificación constituido por las reglas (1)-(5) al definir el orden jurídico $\mathrm{O}_{\mathrm{i}}$ determina su estructura. Esta está dada por las reglas (2)-(5). Las reglas (2), (3) y (4) condicionan el carácter dinámico del orden, mientras que la regla (5) es responsable del carácter sistemático de los sucesivos conjuntos de normas (sistemas). La regla (1) es la que identifica el orden considerado; otros órdenes se distinguen de $\mathrm{O}_{\mathrm{i}}$. no por su estructura (que es idéntica), sino por ser distinta su primera constitución, es decir, por la regla (1). Esta regla no fija ninguna condición especial que deba reunir la primera constitución: cualquier conjunto de normas (que contenga por lo menos una norma de competencia) puede ocupar la posición de una primera constitución y dar lugar a un orden jurídico. (Por supuesto, no todos los órdenes jurídicos son igualmente interesantes; a veces los juristas están interesados en aquel orden jurídico que es efectivo o vigente en una sociedad dada en un tiempo determinado. Pero éste es otro problema.)

El criterio de identificación, tal como fue esbozado aquí, más que una definición de un orden específico $\mathrm{O}_{\mathrm{i}}$, es un esquema definicional. Para ser una definición la regla (1) tendría que especificar concretamente qué normas forman parte de la primera constitución. Ya sabemos que éste es el único punto en que la definición de un orden $\mathrm{O}_{\mathrm{i}}$, difiere de la del orden $\mathrm{O}_{\mathrm{i}}$. Esto permite distinguir entre el criterio de identificación genérico (esquema 
definicional) que determina la estructura de los órdenes jurídicos, y la definición específica de un orden determinado.

La observación de Caracciolo de que «...el criterio de legalidad $\mathrm{C}_{2}$ no suministra, en verdad, condiciones de pertenencia de normas a un conjunto momentáneo, sino que define la pertenencia de conjuntos de normas al orden jurídico» resulta exagerada. En realidad, tal criterio expresado por las reglas (2) y (3) cumple ambas funciones: define la pertenencia de los sistemas al orden y determina (parcialmente) el contenido de cada sistema.

Efectivamente, el contenido de cada sistema perteneciente al orden en cuestión está determinado (en parte) por las reglas (1)-(5), que constituyen, conjuntamente, una definición recursiva de «norma válida en un sistema, $\mathrm{S}_{\mathrm{t}}$ del orden $\mathrm{O}_{\mathrm{i}}$ », en el sentido de que por aplicación sucesiva de esas reglas se puede establecer en un número finito de pasos si una determinada norma pertenece o no a un determinado sistema del orden considerado. Los criterios de pertenencia de una norma a un sistema son tres: a) criterio de pertenencia directa de las normas independientes (regla 1), b) criterio de deducibilidad (regla 5) y c) criterio de legalidad (reglas 2, 3 y 4).

Desde luego, el contenido de un sistema determinado dependerá de los actos normativos (actos de promulgación y derogación) que realicen las autoridades competentes, lo que es una cuestión empírica. Pero la cuestión de saber qué normas son agregadas al sistema como consecuencia de un acto de promulgación y qué normas son eliminadas como consecuencia de un acto de derogación plantea delicados problemas lógicos, que no voy a considerar aquí (cfr. Alchourrón-Bulygin, 1979 y 1981).

La noción de orden jurídico esbozada aquí refleja un uso de esta expresión. Para este concepto la identidad del orden reposa en la continuidad de los sistemas que a él pertenecen y esto quiere decir, en última instancia, la continuidad de la constitución, lo que no implica su inmutabilidad, sino la legalidad del cambio. Todo cambio ilegal de la constitución, es decir, toda revolución jurídica conduce a la ruptura del orden jurídico y la nueva constitución dará origen a un nuevo orden. Así no sólo la revolución rusa de 1917, sino también la promulgación de la Constitución española de 1978 y la restauración de la Constitución argentina en 1983 y cualquier golpe de Estado dan lugar a un nuevo orden jurídico. En este sentido, «orden jurídico» así definido no se identifica con «derecho estatal» o «derecho nacional». En cierto sentido, el orden jurídico del régimen franquista forma parte del derecho español, al igual que el orden constitucional de 1978. Es que los usos lingüísticos son en este punto considerablemente 
vagos y es posible que el concepto de derecho nacional tenga que ser reconstruido como un conjunto de órdenes jurídicos sucesivos (cfr. Caracciolo, 1988, 19-20). Pero este problema excede los límites del presente trabajo.

\section{Pertenencia y aplicabilidad}

He señalado en otro lugar (Bulygin, 1982) que se suele exagerar la importancia de la noción de sistema momentáneo global como conjunto de todas las normas que pertenecen al sistema correspondiente a un momento temporal determinado. La idea es que para un observador (un juez o un jurista) situado en el momento $t$ sólo son aplicables las normas que pertenecen al sistema correspondiente a $t\left(\mathrm{~S}_{\mathrm{t}}\right)$. Esto, sin embargo, es un error. Con mucha frecuencia suelen resultar aplicables normas que pertenecen a algún sistema anterior $\left(\mathrm{S}_{\mathrm{t}-\mathrm{n}}\right)$ y que fueron derogadas antes de t. El concepto de aplicabilidad debe ser cuidadosamente distinguido del de pertenencia. A qué casos es aplicable una norma suele estar determinado por lo que he llamado criterios de aplicabilidad. La regla de la ley más benigna en derecho penal es un ejemplo de tales criterios de aplicabilidad. En virtud de esta regla, el juez debe aplicar la ley penal más benigna con independencia de la cuestión de si tal ley sea vigente o no, es decir, si pertenece o no al sistema correspondiente al momento en que el juez dicta su sentencia.

La distinción entre pertenencia y aplicabilidad de las normas permite diferenciar entre lo que he llamado tiempo externo y tiempo interno de una norma. El tiempo externo es una función de la pertenencia: es el conjunto de los momentos temporales en los que la norma en cuestión pertenece a algún sistema del orden considerado. Si por «existencia» de una norma entendemos su pertenencia a un sistema, podemos decir también que el tiempo externo es el conjunto de los intervalos en los que la norma existe. La existencia (en este sentido) de una norma no tiene por qué ser continua: la misma norma puede pertenecer al sistema $S_{t}$, no pertenecer al sistema $S_{t+1}$, y volver a pertenecer a $S_{t+2}$.

El tiempo interno de una norma puede ser definido como el conjunto de todos los intervalos temporales en los que la norma es aplicable. El tiempo interno es, por lo tanto, una función de aplicabilidad. Los dos tiempos no tienen por qué coincidir: una norma puede pertenecer al sistema $S_{t}$ sin ser aplicable en el momento t y viceversa, puede ser aplicable en el momento $t$ sin pertenecer al sistema $S_{t}$ correspondiente a ese momento. 
De lo dicho se desprende que las normas que un juez aplica a un caso en un determinado momento no necesariamente tienen que pertenecer al sistema correspondiente a ese momento. De hecho, las normas que los jueces aplican para resolver un caso suelen pertenecer a sistemas diferentes del mismo orden jurídico y, a veces, puede tratarse de normas pertenecientes a otros órdenes jurídicos. (Hay toda una disciplina jurídica -Derecho Internacional Privado- que estudia los casos en los que procede la aplicación del derecho extranjero.) Lo que el juez hace efectivamente es determinar -siguiendo los criterios de aplicabilidad- qué normas son aplicables al caso y luego construye un sistema en base a esas normas, que con frecuencia son una selección de sistemas que corresponden a diferentes momentos temporales. Hay, sin embargo, un sentido en que cabe decir que el sistema correspondiente al momento en que el juez ha de tomar su decisión es efectivamente un sistema privilegiado: los criterios de aplicabilidad de las normas que el juez usa tienen que pertenecer a ese sistema. En otras palabras, el juez debe usar los criterios de aplicabilidad vigentes, si bien esos criterios de aplicabilidad pueden remitirlo luego a normas pertenecientes a sistemas anteriores, ya derogadas, e incluso a normas pertenencientes a otros órdenes jurídicos (cfr., Bulygin, 1982).

Puede suceder incluso que una norma sea aplicable a un determinado caso, a pesar de que esa norma no pertenezca a ningún sistema del orden jurídico en cuestión y tampoco a ningún sistema de ningún otro orden jurídico. Esto sucede, por ejemplo, con una ley inconstitucional, es decir, una ley promulgada por una autoridad incompetente. (La autoridad puede ser incompetente por tres motivos distintos: a) por tratarse de un órgano distinto del autorizado, b) por no haberse seguido el procedimiento adecuado y c) por entrar la norma promulgada en conflicto con algún principio o garantía constitucional; este último es el caso más frecuente.) Y sin embargo, una ley de estas características puede ser aplicable, por ejemplo, si el Tribunal Constitucional la declaró constitucional. Ahora bien, la constitucionalidad de una ley no depende de lo que diga el Tribunal Constitucional y una ley que no fue dictada por una autoridad competente sigue siendo inconstitucional, aunque el Tribunal diga lo contrario. Pero es el pronunciamiento del Tribunal Constitucional el que determina la aplicabilidad de la ley. Si el Tribunal dice (erróneamente) que la ley es constitucional, la ley será aplicable, aunque no sea válida en el sistema, Esta tesis, que puede parecer chocante a muchos juristas, es, sin embargo, mera consecuencia lógica de la definición de pertenencia de las normas 
al sistema o validez en el sistema. Una norma pertenece a un sistema o es válida en él, si, y sólo si, ha sido creada por la autoridad competente y no cuando alguien diga que ha sido creada por la autoridad competente, aunque ese «alguien» sea el tribunal de última instancia, competente para decidir acerca de la constitucionalidad de las leyes.

\section{Regla de reconocimiento como criterio de identificación}

Es importante tener presente que las reglas (1)-(5) que constituyen un criterio de identificación de un orden jurídico son reglas conceptuales, es decir, reglas que regulan el uso de un concepto y no normas de conducta. Por «normas de conducta» entiendo normas que prohíben, permiten $\mathrm{u}$ ordenan ciertas conductas o estados de cosas resultantes de las conductas. Las reglas conceptuales, en cambio, no prohíben ni permiten nada; las reglas matemáticas, las reglas lógicas, las reglas del lenguaje (sintácticas y semánticas) son típicos ejemplos de reglas conceptuales.

Una de las tesis principales de este trabajo es que la unidad de un orden jurídico y de cada uno de los sistemas que pertenecen a él está constituida por una regla conceptual (criterio de identificación) y no por una norma de conducta. Filósofos del derecho tan importantes como Kelsen y Hart no parecen compartir esta idea. Para Kelsen la unidad del orden jurídico está dada por la norma básica o fundamental y para Hart por la regla de reconocimiento. Cabe preguntarse si la norma básica de Kelsen y la regla de reconocimiento de Hart son genuinas normas de conducta o reglas conceptuales. No voy a analizar aquí la teoría de la norma básica de Kelsen. En otro lugar (Bulygin, 1990) he analizado detenidamente la cuestión y he llegado a la conclusión que la norma básica no es necesaria para la definición del orden jurídico y no es suficiente para fundamentar la pregunta por la obligatoriedad de las normas jurídicas. Por lo tanto, me limitaré aquí al análisis de la regla de reconocimiento de Hart.

En un artículo publicado hace muchos años (Bulygin, 1976) sostuve que la regla de reconocimiento de Hart ha de interpretarse como una regla conceptual y no como una norma, porque tiene carácter definicional y carece de todo contenido normativo. En particular, esa regla no prescribe a los jueces el deber de aplicar las normas jurídicas y tal deber -cuando lo hay, lo que es una cuestión contingente- surge no de la regla de reconocimiento, 
sino de alguna norma específica del sistema, identificada conforme a la regla de reconocimiento.

No era mi intención, desde luego, hacer una exégesis del pensamiento de Hart; no cabe duda de que Hart mismo y prácticamente todos sus comentaristas, entre los cuales figuran tan profundos conocedores de su obra como Raz (1971). Hacker (1977) y MacCormick (1978 y 1981), piensan que la regla de reconocimiento es una genuina norma que prescribe a los jueces el deber de aplicar las normas jurídicas identificadas por esa regla. Mi pretensión era ofrecer una interpretación alternativa de la regla de reconocimiento (con algún apoyo en The Concept of $L a w$ ) que explique mejor su funcionamiento y el papel que desempeña en la dinámica jurídica. Para ello ofrecí algunos criterios de distinción entre reglas conceptuales y normas de conducta. Las normas de conducta establecen deberes y prohibiciones; respecto de ellas tiene sentido hablar de obediencia o desobediencia, mientras que las reglas conceptuales se limitan a definir un concepto, no establecen prohibiciones ni obligaciones y carece de sentido hablar de obediencia o desobediencia a tales reglas. El no uso (o mal uso) de las reglas conceptuales puede dar lugar a reacciones críticas, pero éstas son de una naturaleza muy distinta que las que provoca el incumplimiento de una norma de conducta. Lo que se reprocha al que no sigue o no usa reglas conceptuales es ignorancia y no desobediencia. Piénsese en la persona que realiza una jugada incorrecta en ajedrez (por ejemplo, enroca, estando bajo jaque) o que no sabe usar correctamente las reglas gramaticales o matemáticas. En ninguno de estos casos diríamos que la persona en cuestión ha desobedecido las reglas de ajedrez, de gramática o de matemática; diríamos simplemente que no sabe jugar al ajedrez, hablar correctamente el idioma en cuestión o hacer cálculos matemáticos.

Recientemente, mi tesis ha sido objeto de una aguda crítica por parte de Juan Ruiz Manero en un muy interesante libro de próxima aparición (Ruiz Manero). A pesar de la crítica de Ruiz Manero, hoy -catorce años después de la publicación de mi artículo- estoy dispuesto a defender una tesis si no idéntica, al menos muy similar. Como se verá a continuación, la tesis que trataré de defender no se limita a la interpretación de la regla de reconocimiento hartiana, sino que tiene un alcance más general: sostengo que sin un criterio conceptual que permita identificar las normas del sistema, no se puede hablar de deberes que establezcan tales normas. Mi diferencia con Hart y con Ruiz Manero puede sintetizarse así: ellos creen que primero tenemos una norma que obliga a aplicar las normas que reúnen ciertas características 
y luego, de esta norma se extrae el criterio conceptual que define qué normas pertenecen al sistema; yo, en cambio, sostengo que para poder determinar qué deberes establecen las normas jurídicas, hay que tener primero un criterio conceptual que nos permita identificar tales normas.

En el artículo de 1976 sostuve las dos tesis siguientes: 1) la regla de reconocimiento como conjunto de los criterios de identificación del derecho válido establece qué propiedades debe reunir una norma para ser considerada derecho válido, y 2) cuando los jueces tienen el deber de aplicar el derecho, tal deber no surge de la regla de reconocimiento (entendida como criterio de identificación a la manera de las cinco reglas esbozadas en la sección IV), sino de alguna norma jurídica, identificada como válida conforme a la regla de reconocimiento.

Dos son los cargos que me formula Ruiz Manero: «...primero, que en la caracterización de la regla de reconocimiento y en su consideración de la misma como regla conceptual hay una confusión entre la propia regla de reconocimiento (como norma o regla de conducta aceptada por los jueces) y el criterio de identificación de las reglas del sistema basado en la regla de reconocimiento (como criterio usado por un observador externo, como pueda ser, por ejemplo, un teórico del Derecho o un consejero jurídico privado) y, segundo, que la consideración, propuesta por Bulygin, de la regla de reconocimiento como regla conceptual nos deja impotentes para entender la naturaleza y la fuente del deber judicial de decidir conforme a las normas válidas.»

El primer cargo me parece totalmente injusto; en ningún momento confundí la regla de reconocimiento como norma de conducta aceptada por los jueces que establece el deber de aplicar las normas jurídicas que reúnen los requisitos que esa regla fija con el criterio de identificación de las reglas del sistema basado en tal regla de reconocimiento. Lo que hice era muy distinto: propuse llamar «regla de reconocimiento» al criterio de identificación (cfr. Bulygin, 1976, pág. 35: «Llamaremos regla de reconocimiento al conjunto de todos los criterios de identificación...»). Formulé esta propuesta porque consideraba y sigo considerando que hace falta usar un criterio de identificación para poder identificar las normas jurídicas válidas y porque el deber de los jueces de aplicar las normas jurídicas válidas proviene de esas mismas normas, identificadas conforme a ese criterio. En otras palabras, creo que primero viene el criterio de identificación (regla conceptual) y luego las normas de conducta que establecen deberes, mientras que Ruiz Manero y los comentaristas de Hart, como Raz, Hacker y MacCormick (y probablemente 
también Hart mismo) creen poder derivar el criterio de identificación de una norma de conducta. Puedo tener razón o no, pero no hay, ni hubo aquí confusión alguna de mi parte.

El segundo cargo es mucho más serio. Si mi interpretación de la regla de reconocimiento nos dejara efectivamente impotentes para entender la fuente del deber judicial de decidir conforme a las normas válidas, mí posición se derrumbaría. Pero no creo que suceda tal cosa; mi interpretación de la regla de reconocimiento como criterio conceptual no impide en modo alguno dar una explicación coherente del deber judicial. Creo, en cambio, que la interpretación estándar de la regla de reconocimiento nos deja en la más absoluta ignorancia, pues no sólo no podemos saber cuáles son los deberes jurídicos de los jueces, si no hemos identificado previamente las normas jurídicas, sino ni siquiera estamos en condiciones de identificar a los jueces. Pero veamos primero el argumento de Ruiz Manero. Para evitar malos entendidos lo transcribiré in extenso:

«La aparente plausibilidad de la presentación que hace Bulygin de la fuente del deber del juez se desmorona si analizamos qué puede querer decir el juez cuando dice que "conforme al Derecho, debo castigar a este hombre, pero, por razones morales, no lo voy a hacer». Este «debo» no significa, obviamente, «moralmente debo» (y por ello el juez puede decir sin contradicción que conforme al Derecho debe hacer $\mathrm{x}$, pero que por razones morales no debe hacerlo). Ello excluye que el deber al que se refiere el juez de aplicar la norma que le ordena condenar al acusado surja de que lo ordenado por tal norma sea moralmente correcto y también que ese deber surja de que sea moralmente correcto obedecer, aplicándolas, a las normas dictadas por una determinada autoridad (en ambos casos, el juez no podría decir sin contradicción «conforme al Derecho debo..., pero por razones morales no debo...»). Pero si el deber del juez de aplicar la norma que le ordena condenar surgiera -como pretende Bulygin- de esa misma norma (a la que llamaremos $\mathrm{N}_{1}$ ), ello sólo podría tener como fundamento el que lo ordenado por esa norma $\mathrm{N}_{1}$ es moralmente correcto. En otro caso, es preciso, para fundamentar ese deber, apelar a una norma $\left(\mathrm{N}_{2}\right)$ distinta de la que se aplica que ordene al juez aplicar $\mathrm{N}_{1}$ y entonces "conforme al Derecho, debo aplicar $\mathrm{N}_{1}$ " significaría "hay una norma $\mathrm{N}_{2}$ que me ordena aplicar $\mathrm{N}_{1}$ ". A su vez, y para salvaguardar la posibilidad de que el juez pueda decir sin contradicción "conforme al Derecho (es decir, conforme a $\mathrm{N}_{2}$ ) debo aplicar $\mathrm{N}_{1}$ pero por razones morales no debo", $\mathrm{N}_{2}$ no puede ser una norma moral que estatuya un deber moral de aplicar $\mathrm{N}_{1}$ : esto es, el 
deber a que se refiere el juez no puede tener como fundamento una $\mathrm{N}_{2}$ de naturaleza moral. $\mathrm{N}_{2}$, por consiguiente, no puede ser una norma moral. Y dada la irrelevancia, en este contexto, de otros códigos normativos (tales como las reglas de etiqueta, las del buen gastrónomo $\mathrm{u}$ otros códigos semejantes que pudieran imaginarse) no parece problemático afirmar que, en tal caso, $\mathrm{N}_{2}$ sólo puede ser una norma jurídica. $\mathrm{Y}$, entonces, quedan dos posibilidades: o bien $\mathrm{N}_{2}$ es una norma jurídica derivada -esto es, una norma que pertenece al sistema en virtud de su concordancia con los criterios de validez jurídica establecidos por otra norma- o bien $\mathrm{N}_{2}$ es una norma jurídica última (en el sentido visto en el $\S$ II, 1). Y eso es precisamente la regla de reconocimiento: una norma jurídica que establece cuáles son los criterios últimos de validez jurídica, esto es, una norma que establece, con carácter último, cuáles son las normas que los jueces tienen el deber de aplicar. Obviamente, esta regla jurídicamente última puede considerarse ubicada, por las diversas teorías del Derecho, bien en algún momento de la creación del Derecho (como es el caso de la norma básica kelseniana) bien en el momento de la aplicación (como es el caso de la regla de reconocimiento de Hart). Pero esto no afecta a la presente discusión, pues Bulygin concuerda con la orientación hartiana de considerar como criterio último de identificación del "Derecho válido" el efectivamente usado por los órganos de aplicación; y lo que aquí se ha tratado de demostrar es que, para fundamentar la afirmación de que los jueces tienen el deber de aplicar el "Derecho válido" y que este deber es de naturaleza no-moral, hay que entender ese criterio de identificación del "Derecho válido" efectivamente usado por los jueces no como un criterio meramente definicional, relativo al uso de la expresión "Derecho válido", sino como una norma, que establezca, para los jueces, el deber de aplicar las normas que lo satisfagan; en definitiva, que para poder dar cuenta del deber de los jueces de aplicar el Derecho como un deber de naturaleza no moral, la regla de reconocimiento ha de ser entendida como la entiende Hart, esto es, como una norma genuina y no, como pretende Bulygin, como una mera regla definicional.» (Ruiz Manero, Capítulo Segundo, III.1).

Creo que no cometeré mayores injusticias con Ruiz Manero, si resumo su argumento del siguiente modo:

El código penal ordena al juez condenar al que comete homicidio, pero frente a la pregunta «¿por qué debe el juez aplicar el código penal?», caben dos respuestas: a) porque es moralmente correcto, y b) porque hay una norma jurídica que ordena aplicar el código penal. La respuesta a) queda descartada (porque en tal 
caso el juez no podría decir sin contradicción «debo jurídicamente, pero no debo moralmente»). La respuesta b) nos lleva a otra norma (que por las mismas razones tiene que ser jurídica y no moral); esta segunda norma podrá ser derivada (en cuyo caso nos remitirá a otra norma jurídica), pero tarde o temprano tenemos que llegar a una norma jurídica última, y ésta es la regla de reconocimiento.

Ahora bien, la idea de una norma jurídica última es más que dudosa. Una vez que comenzamos a preguntar por el fundamento de las normas, ¿por qué no seguir preguntando? -Qué nos impide preguntar por el fundamento de esa norma jurídica que Ruiz Manero califica como última (y que dejaría de ser última al formularse la pregunta por su fundamento)?

Esto me hace recordar la historia de la secretaria a quien su jefe solía dejar por escrito las instrucciones para el trabajo del día. Un día la secretaria llega a la oficina y se pregunta: «¿Qué debo hacer?» Encuentra la orden del jefe que dice «¡Copie este manuscrito!», pero en vez de hacerlo, se vuelve a preguntar: «¿Por qué debo obedecer las órdenes de mi jefe?» No tarda en encontrar una respuesta: «Porque me han contratado como secretaria y debo cumplir el contrato», pero acto seguido se le ocurre otra pregunta: «¿Por qué debo cumplir el contrato?» La respuesta «porque así lo ordena el Código Civil», tampoco la satisface y se pregunta «¿por qué debo obedecer las normas del Código Civil?» y sigue así durante un buen rato. En el momento en que está por preguntar «¿por qué debo obedecer la primera constitución?», llega el jefe y al ver que la secretaria en vez de copiar el manuscrito pasó la mañana preguntándose qué debe hacer, le dice con tono poco amistoso: «¡Deje de formular preguntas tontas y haga lo que se le ha ordenado!»

La moraleja de esta historia es doble: 1) para justificar una acción no es suficiente mencionar una norma, hay que usarla. La distinción entre uso y mención es fundamental en este contexto. La secretaria hablaba acerca de normas, pero no usó ninguna y por eso no hizo nada. 2) La pregunta «¿por qué debo obedecer esta norma?» nos remite a otra norma, frente a la cual siempre cabe formular la misma pregunta. La serie de preguntas por el fundamento es infinita. Frente a cualquier norma puedo reiterar la pregunta. No se ve por qué la mayoría de los filósofos del derecho de repente se paran frente a ciertas respuestas y no siguen preguntando. ¿Acaso los jueces no pueden preguntar por qué deben obedecer la regla de reconocimiento? Decir, como hace Ruiz Manero, que esta regla es última equivale a la respuesta del jefe: «¡No pregunte más, haga lo que se le ha ordenado!» 
(Tampoco ofrece una respuesta satisfactoria una teoría, como la de Nino, que recurre a normas morales, porque también cabe preguntar «ipor qué debo obedecer las normas morales?») Por lo tanto, si uno quiere saber qué debe hacer, tiene que usar una norma y no preguntar por su fundamento.

Veamos un ejemplo. Se ha probado que Juan ha cometido un homicidio y el juez se pregunta: «¿Qué debo hacer con Juan?»El código penal, uno de cuyos artículos dice «El que matare a otro será penado con prisión de ocho a veinticinco años», le da la respuesta: debe condenarlo a prisión. Además, el código procesal contiene una norma que dice que los jueces deben fundar sus sentencias en el derecho vigente. Usando estas dos normas el juez está en condiciones de resolver su problema: el código penal le dice que debe condenar al homicida y la norma del código procesal le dice que debe invocar el código penal para justificar su decisión. Si en vez de hacerlo, sigue preguntando qué debe hacer, no cumple con sus deberes de juez y se expone al peligro de perder su cargo.

La serie infinita de preguntas por el fundamento de las normas lleva a una parálisis total y la única forma de salir de esta parálisis es dejar de preguntar por el fundamento y usar una norma como pauta de conducta, para lo cual es necesario identificar las normas que interesan en este contexto. Si se trata de un juez, debe usar las normas jurídicas y para identificarlas tiene que recurrir a un criterio conceptual, es decir, a una definición.

Cuando Ruiz Manero afirma «Pero si el deber del juez de aplicar la norma que le ordena condenar surgiera -como pretende Bulygin- de esa misma norma (a la que llamaremos $\mathrm{N}_{1}$ ), ello sólo podría tener como fundamento el que lo ordenado por esa norma $\mathrm{N}_{1}$ es moralmente correcto», no advierte que el juez no tiene ninguna necesidad de preguntar por el fundamento de esa norma; lo que tiene que hacer es usar esa norma que le dice qué debe hacer. Y si bien el juez puede formular la pregunta por el fundamento de $\mathrm{N}_{1}$ con esta pregunta no resuelve su problema. Además, como ya hemos visto, puede formular la misma pregunta frente a $\mathrm{N}_{2}$ y frente a cualquier norma que se invoque como fundamento, sea ésta jurídica, moral o religiosa. Para pasar al plano de la acción, el juez debe dejar de mencionar normas y usar una norma. Y lo que los jueces de hecho hacen es usar las normas jurídicas que ellos identifican de acuerdo a un criterio de identificación compartido, al que he propuesto llamar regla de reconocimiento.

Es obvio que el juez no puede usar normas jurídicas si previamente no las identificó y no puede identificarlas si no dispone 
de un criterio que le diga cuáles son. Por lo tanto, el orden lógico de las cosas es el siguiente: primero el juez tiene que tener un criterio de identificación de las normas jurídicas; luego usando este criterio identifica las normas y son éstas las que le dicen qué debe hacer. Luego actúa (dicta sentencia) y usa las normas para justificar su acción.

\section{Regla de reconocimiento y la definición de «juez»}

Al colocar en el principio una norma que obliga a los jueces a aplicar las normas que reúnen ciertos requisitos (gracias a los que se convierten en normas jurídicas válidas) y al derivar el criterio de identificación de las normas jurídicas de esta norma primera, Hart y sus secuaces se privan no sólo de la posibilidad de decir que la regla de reconocimiento entendida como una norma de conducta sea una norma jurídica válida, sino que, además, no pueden determinar quiénes son jueces. Es que cualquier persona normal identifica a los jueces mediante normas jurídicas (jueces son los individuos nombrados por un procedimiento previsto en la constitución y en las leyes, que tienen la competencia para resolver los conflictos y las controversias mediante la aplicación de las normas jurídicas), y así lo hace también Hart, pero esto convierte su teoría en circular, como se encarga de destacar el propio Ruiz Manero (Capítulo Segundo, II.1):

«En efecto, de acuerdo con Hart, para poder determinar el contenido de la regla de reconocimiento hemos de acudir, como se ha repetido, a los criterios de validez jurídica aceptados y seguidos por los jueces y tribunales; pero -también, según Hart no podemos identificar quiénes son jueces y tribunales sino sobre la base de las reglas de adjudicación del sistema, pues, como él mismo dice, "la existencia de un tribunal implica la existencia de reglas secundarias que confieran potestad jurisdiccional” (CD, pág. 170).»

Los seguidores de Hart han intentado romper este círculo mediante teorías más o menos ingeniosas, pero insatisfactorias, como muestra elocuentemente Ruiz Manero, quien analiza en detalle las propuestas de MacCormick y de Nino. Tras destacar las insuficiencias de estos intentos, formula su propia propuesta que, lamentablemente, es tan poco satisfactoria como las de sus predecesores.

La propuesta de Nino (Nino, 1980) consiste en la caracterización del concepto de juez en términos fácticos: jueces o, como 
dice Nino, órganos primarios son «los que de hecho pueden (en el sentido fáctico y no normativo de la palabra "poder") determinar el ejercicio del monopolio coactivo estatal en casos particulares» (Nino, 1980, pág. 128).

A esta altura debería ser claro que una teoría como la de Nino no puede lograr su propósito por la sencilla razón de que el concepto de juez es un concepto normativo y no se puede definir un concepto normativo en términos puramente fácticos. Todo intento de este tipo está destinado al fracaso.

Ruiz Manero señala con razón que la caracterización fáctica de «juez» que propone Nino «viene a ser una versión aplicada a la judicatura de la caracterización de "legislador" en términos de obediencia habitual que propusieran Bentham y Austin» (Ruiz Manero, Capítulo Segundo, II.2) y es pasible de las mismas críticas que Hart dirigiera con demoledora fuerza a sus ilustres predecesores (cfr. Hart, 1961, cap. IV). Coincido totalmente con Ruiz Manero en este punto. Sólo puedo agregar que la referencia que hace Nino en su definición de «juez» al «monopolio coactivo estatal» puede conducirlo fácilmente al mismo círculo que pretende eludir, pues los conceptos de «estado» y de «monopolio» tienen un fuerte sabor normativo y no sé cómo se las podría arreglar Nino para definirlos en términos puramente fácticos.

La propuesta de MacCormick (1981) va por otros carriles. En lugar de intentar una caracterización fáctica, propone una definición normativa, pero no en términos de normas jurídicas identificadas como válidas conforme a la regla de reconocimiento (en la terminología de Hart, reglas de adjudicación que confieren poder jurisdiccional), sino en términos de reglas sociales de deber. Según MacCormick el concepto de juez puede caracterizarse en términos de normas consuetudinarias que establecen que ciertos individuos a) tienen el deber de juzgar sobre cualquier reclamo o disputa, b) tienen el deber de formular su juicio por referencia a pautas de conducta preexistentes, y c) tienen el monopolio sobre el uso justificado de fuerza en virtud de pautas prevalecientes en esa sociedad.

Ruiz Manero critica esta propuesta, porque considera que el concepto de juez no puede ser suficientemente caracterizado en términos de normas que prescriben deberes; lo que define al «juez» es el poder normativo de decidir las controversias mediante decisiones autoritativas u obligatorias. En esto Ruiz Manero coincide con Carrió, para quien no se puede caracterizar adecuadamente el status normativo de los jueces sin hacer referencia a sus competencias o potestades, inmunidades, sujeciones y deberes (Carrió, 1986). 
En esto Ruiz Manero tiene razón. Pero si éste fuera el único defecto de la teoría de MacCormick, sería fácil mejorarla. Bastaría para ello introducir en la definición de «juez» el elemento de «poder»; las mismas reglas sociales que establecen los deberes del juez, le conferirían también el poder de dictar resoluciones obligatorias. En esto consiste efectivamente la propuesta de Ruiz Manero; él cree poder ofrecer de este modo una caracterización normativa del concepto de juez sin incurrir en circularidad, porque las reglas sociales que confieren poderes y fijan deberes (y de esta manera determinan quiénes son los jueces) no son normas jurídicas válidas, identificadas conforme al criterio extraído de la regla de reconocimiento.

No me parece satisfactoria esta teoría, por varias razones. En primer lugar, la teoría pasa por alto un dato empírico de suma importancia, a saber, que -al menos en los órdenes jurídicos desarrollados o maduros- existen un gran número de normas legisladas (algunas de rango constitucional, otras legales) que regulan minuciosamente el status normativo de los jueces (cfr. Carrió, 1986) y que los juristas (y los simples mortales) usan esas normas para identificar a los jueces. Y como esas normas son normas identificadas como válidas, se sigue que si los criterios de validez surgen de la regla de reconocimiento, volvemos a caer en la circularidad: para determinar quiénes son jueces tenemos que usar la regla de reconocimiento y para identificar la regla de reconocimiento tenemos que saber quiénes son jueces. De modo que la teoría MacCormick-Ruiz Manero resulta si no empíricamente falsa, al menos altamente artificiosa.

En segundo lugar, una teoría del derecho que deja fuera del derecho a gran número de normas que normalmente son consideradas normas jurídicas no puede considerarse satisfactoria. En efecto, las reglas sociales a las que recurren MacCormick y Ruiz Manero para caracterizar normativamente la noción de juez no son normas que integren el sistema jurídico (Ruiz Manero reconoce que se trata de una caracterización extrasistemática); por lo tanto, no son normas jurídicas. Si ya es un grave inconveniente teórico de la teoría de Hart el que el sistema jurídico está basado en una norma no jurídica (la regla de reconocimiento última), este inconveniente se multiplica en la teoría de Ruiz Manero, en la que el sistema jurídico presupone una variedad de normas no jurídicas (llamadas reglas sociales), con el agravante de que esas normas son pacíficamente consideradas por los juristas como normas jurídicas genuinas.

En tercer lugar, es fácil advertir que Ruiz Manero recurre, para escapar a la circularidad, a un simple truco verbal: llama 
«reglas sociales» a aquellas normas que determinan el status normativo del juez y que el resto de los mortales llama «normas jurídicas». Pero un cambio de nombre no es una solución satisfactoria para un problema conceptual.

Todas estas dificultades desaparecen como por arte de magia una vez que reconozcamos que no hay deberes jurídicos que no sean establecidos por normas jurídicas y que para saber cuáles son las normas jurídicas tenemos que disponer de un criterio de identificación (una definición) de tales normas. Por eso he propuesto que la regla de reconocimiento de Hart sea interpretada no como una norma que establece deberes a los jueces, sino como una regla conceptual que fija los criterios de identificación de un orden jurídico y de las normas que pertenecen a los diversos sistemas de ese orden. Desde luego, puede haber distintos criterios. Distintas personas puede usar distintas definiciones (de hecho las definiciones van a variar en la cláusula [1]), es decir, respecto de la constitución que se adopte como punto de partida), pero sólo habrá un orden jurídico, cuando la población en general y, sobre todo, los jueces y otros órganos oficiales compartan la misma definición: la unidad del orden jurídico depende del hecho de que una y la misma definición sea efectivamente usada en un grupo social.

\section{BIBLIOGRAFÍA}

Alchourrón-Bulygin(1971), Carlos E. Alchourrón and Eugenio Bulygin, Normative Systems, Springer, Viena-Nueva York, 1971.

(1976), «Sobre el concepto de orden jurídico», 8, Crítica, 3-23.

(1979), Sobre la existencia de las normas jurídicas, Universidad de Carabobo, Valencia (Venezuela).

(1981), «The expressive conception of nomis», en R. Hilpinen (ed.), New Studies in Deontic Logic,

Reidel, Dordrecht.

(1982), «Definiciones y normas», en Bulygln-Farrell-Nino-Rabossi (comps.), El lenguaje del Derecho. Homenaje a Genaro R. Carrió, Abeledo-Perrot, Buenos Aires.

Bulygin(1976), Eugenio Bulygin, «Sobre la regla de reconocimiento», en AA.VV., Derecho, Filosofía y Lenguaje. Homenaje a Ambrosio L. Gioja, Astrea, Buenos Aires. 
- (1982), «Time and Validity», en A. Martino (ed.), Deontic Logic, Computational Linguistics and Legal Information Systems, North Holland, Ainsterdam-Nueva York-Oxford.

- (1986), «Legal dogmatics and the systematization of law», Reclustheorie, Belheft, 10, 193-210.

- (1990), «An Antinomy in Kelsen's Pure Theory of Law», 3 Ratio Juris, 29-45.

Caracciolo (1988), Ricardo A. Caracciolo, El sistema jurídico. Problemas actuales, Centro de Estudios Constitucionales, Madrid.

Carrió (1986), Genaro R. Carrió, «Sobre los jueces y su status normativo», en M. Laclau y D.

Cracogna (comps.), Teoría General del Derecho. Sus problemas actuales. Estudios en homenaje a Julio C. Cueto Rúa, Hellastra, Buenos Aires.

Hacker (1977), P. M. S. Hacker, «Harcs philosophy of law», en Hacker-Raz (eds.) Law, Morality, and Society. Essays in honour of H. L. A. Hart, Clarendon Press, Oxford.

Hart (1961), H. L. A. Hart, The Concept of Law, Clarendon Press. Oxford.

MacCormick (1978), Neil D. MacCormick, Legal Reasoning and Legal Theory, Clarendon Press, Oxford.

- (1981), H. L. A. Hart, Edward Arnold Publishers, Londres.

Nino (1980), Carlos S. Nino, Introducción al análisis del Derecho, Astrea, Buenos Aires.

Raz (1970), Joseph Raz, The Concept of a Legal System, Clarendon Press, Oxford.

- (1971), «The Identity of Legal Systems», 59 California Law Review, 795-815 (reproducido en J. Raz, The Authoriti, of Law, Clarendon Press, Oxford, 1979).

Ruiz Manero, Juan Ruiz Manero, Jurisdicción y normas. Dos estudios sobre función jurisdiccional $y$ teoría del Derecho (en prensa).

Tarski (1956), Alfred Tarski, Logic, Semantics, Metamathematics, Clarendon Press, Oxford. 